\title{
Review Article \\ Gastrodia elata Blume (Tianma): Hope for Brain Aging and Dementia
}

\author{
Klaus Heese \\ Graduate School of Biomedical Science and Engineering, Hanyang University, 222 Wangsimni-ro, Seongdong-gu, \\ Seoul 133791, Republic of Korea \\ Correspondence should be addressed to Klaus Heese; klaus@hanyang.ac.kr
}

Received 19 August 2020; Revised 26 October 2020; Accepted 3 November 2020; Published 28 December 2020

Academic Editor: Yong Wang

Copyright ( $\odot 2020$ Klaus Heese. This is an open access article distributed under the Creative Commons Attribution License, which permits unrestricted use, distribution, and reproduction in any medium, provided the original work is properly cited.

Since aging-related diseases, including dementia, represent major public health threats to our society, physician-scientists must develop innovative, interdisciplinary strategies to open new avenues for development of alternative therapies. One such novel approach may lie in traditional Chinese medicine (TCM). Gastrodia elata Blume (G. elata, tianma) is a TCM frequently used for treatment of cerebrocardiovascular diseases (CCVDs). Recent studies of G. elata-based treatment modalities, which have investigated its pharmacologically relevant activity, potential efficacy, and safety, have employed G. elata in well-characterized, aging-related disease models, with a focus on models of aging-related dementia, such as Alzheimer's disease (AD). Here, I examine results from previous studies of $G$. elata, as well as related herbal preparations and pure natural products, as prophylaxis and remedies for aging-related CCVDs and dementia. Concluding, data suggest that tianma treatment may be used as a promising complementary therapy for $\mathrm{AD}$.

\section{Introduction}

Aging-related dementia, which is mediated by damage to brain cells induced by pathways, such as those underlying Alzheimer's disease (AD), cerebrocardiovascular diseases (CCVDs), and other neurodegenerative diseases (NDs), is causing great inquietude, anxiety, and discomposure in an aging society [1-7]. The World Health Organization (WHO) has recognized the imperative for globally coordinated research to combat dementia [8]. Much hope has been based on use of stem cell-based therapies; however, such approaches still have to overcome major challenges [9].

Thus, with dementia posing a health threat to elderly people, social awareness of healthy lifestyle choices that can prevent aging-related neuroinflammation and cognitive dysfunction has been attracting increasing attention. In particular, a healthy diet, exercise, and caloric restriction have been demonstrated to be preventive against new-onset $\mathrm{AD}$ and to effectively ameliorate the symptoms of $\mathrm{AD}[10,11]$. Familial (early-onset, younger than 65 years) AD is caused by genetic mutations [12-15]. However, the majority of AD cases
( $95 \%)$ is the sporadic non-inherited form, which is also referred to as late-onset (non-familial, sporadic) AD $[14,16]$. Sporadic AD is likely caused by normal aging $[16,17]$ and its associated consequences, including oxidative stress and disturbance of protein homeostasis [13, 18-20].

Recently, many companies have stopped their AD-related clinical trials and minimized their investments in neurological studies [21]. Therefore, we need new approaches to open doors for alternative therapeutic strategies against aging-related NDs and dementia. In the past few years, alternative medicine has come into focus for the potential to provide new therapeutic measures for dementia [22-25]. Recent comparative proteomics research studies regarding $\mathrm{AD}$-related TCM treatments revealed novel data that suggest that potential mechanisms of action of TCM for the prevention of $\mathrm{AD}$ pathogenesis involve improving the ubiquitin proteasome system (UPS, including chaperones and cochaperones (notably, heat shock proteins (HSPs) and FK506 binding proteins (FKBPs))) [20, 26]. Particularly, G. elata (tianma) received special attention and will therefore be discussed in more detail as follows [26]. 


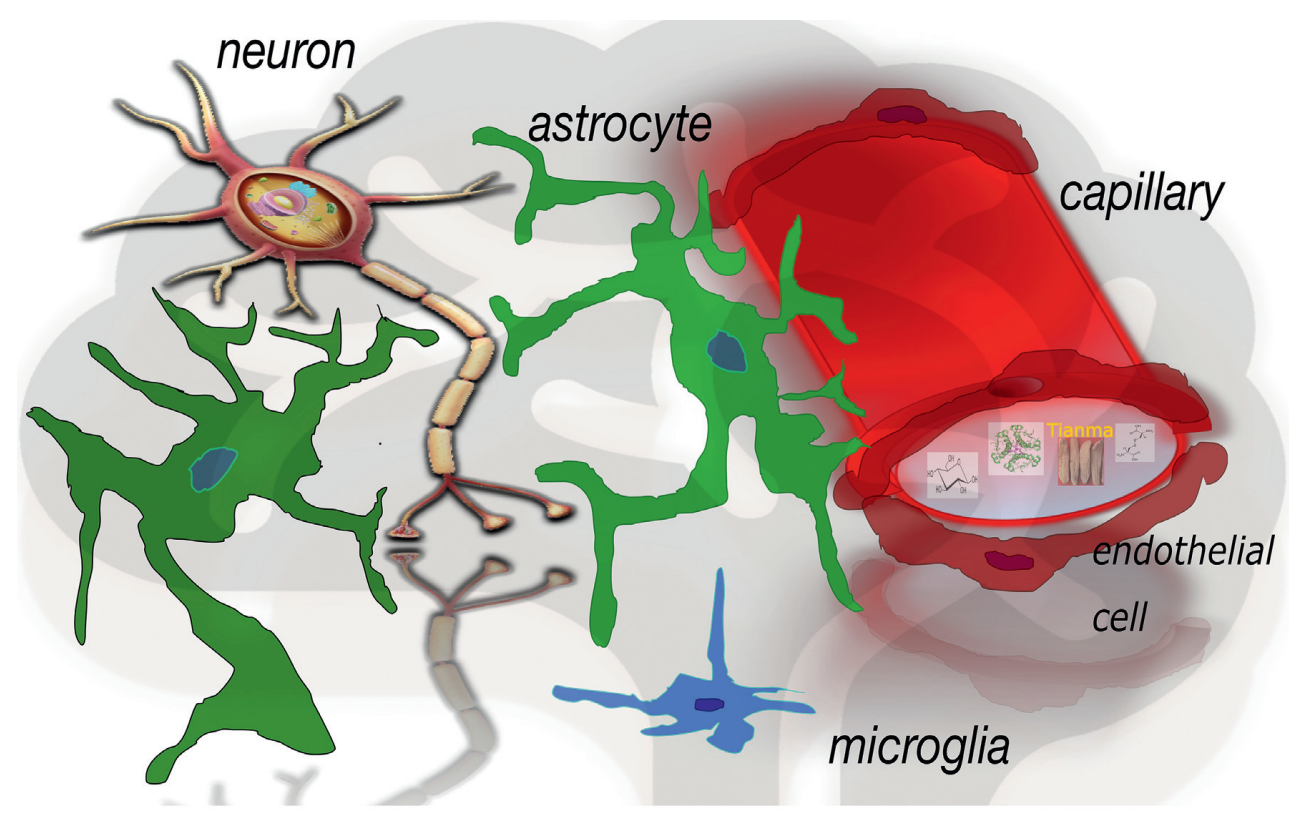

(a)
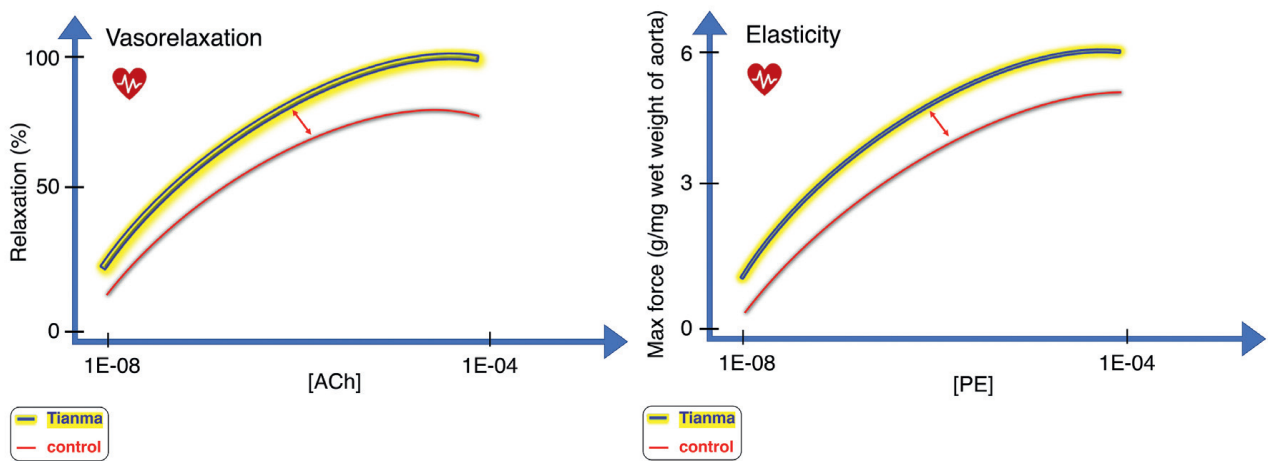

(b)

(c)

FIGURE 1: Schematic overview of tianma-mediated CCV-related activities. (a) With astrocytes serving as sensors and mediators between neural signal transmission and the vascular-dependent energy (glucose) supply, tianma can improve vascular activities and, upon uptake into the cerebrum, neuronal activities and survival and provide neuroprotection against ischemic strikes [33, 41]. (b) Quantitative data showing tianma-enhanced vasorelaxation. Elderly rats were treated with tianma for a period of three months $(\sim 2.5 \mathrm{~g} / \mathrm{kg} / \mathrm{day})$, after which their thoracic aortas were isolated. Dose-response analysis with increasing level of acetylcholine- (ACh-) induced relaxation in $\mathrm{KCl}(80 \mathrm{mM})$ or phenylephrine (PE, $\left.10^{-6} \mathrm{M}\right)$ precontracted isolated endothelium-intact arterial rings [67]. (c) Quantitative representation of tianmaincreased vascular contractile force and elasticity. Dose-response comparison of maximum contractile force in response to increasing concentration of PE in endothelium-intact thoracic aortic rings (preincubated with $80 \mathrm{mM} \mathrm{K}^{+}$) in tianma-treated elderly rats and controls [67].

\section{G. Elata (Tianma) and NDs}

G. elata (tianma) is a member of the Orchidaceae family and has its origin in East Asia. Its tuber has been used in TCM for centuries [26-30], and extracts of tianma or its active ingredients convey physiological- and health-promoting features, including antitumor, memory improving, and neuroprotective activities [30-33]. Particularly, this TCM has been widely used in Asia to treat dizziness, paralysis, epilepsy [34], and hypertension [35]. Tianma has also been used in this region to overcome cognitive deficits and prevent NDs [30, 36-41], including AD [42-46], vascular dementia (VD) [33, 41], and Parkinson's disease (PD)
$[47,48]$, with gastrodin and 4-hydroxybenzyl alcohol among the primary active components [48-53].

\section{Tianma Mobilizes the Cerebrocardiovascular System}

It is common knowledge that heart health contributed to brain health. Connections between AD, VD, diabetes mellitus (type 2, T2DM), and CCVDs have been proposed based on the strong associations between cardiovascular risk factors and $\mathrm{AD}$ and $\mathrm{VD}$, suggesting that these diseases share common characteristics [54-57]. The risk of developing aging-related $\mathrm{AD}, \mathrm{VD}$, and CCVDs appears to 


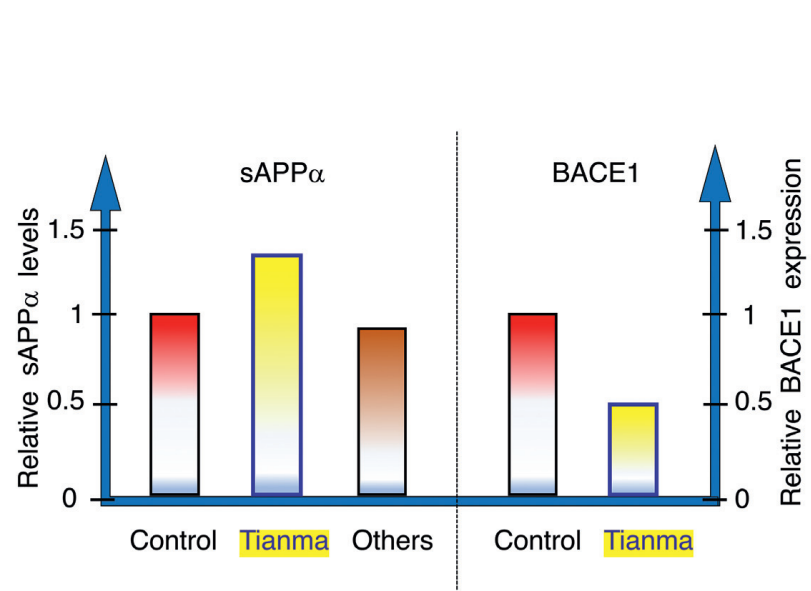

(a)

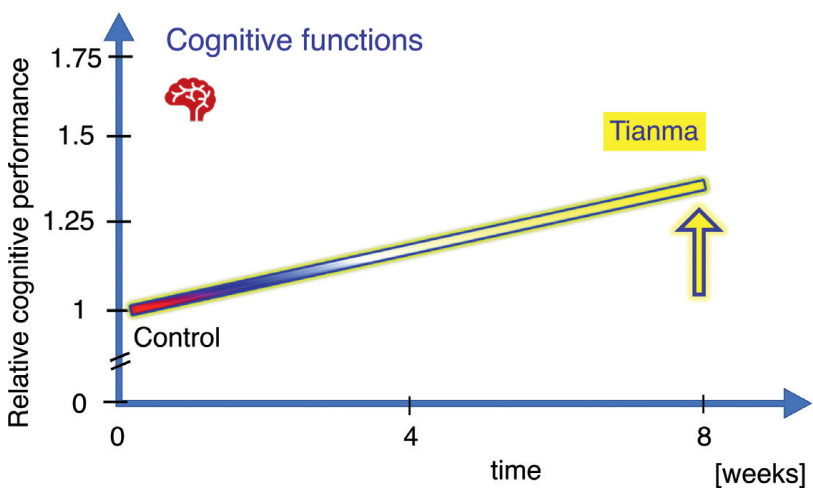

(c)

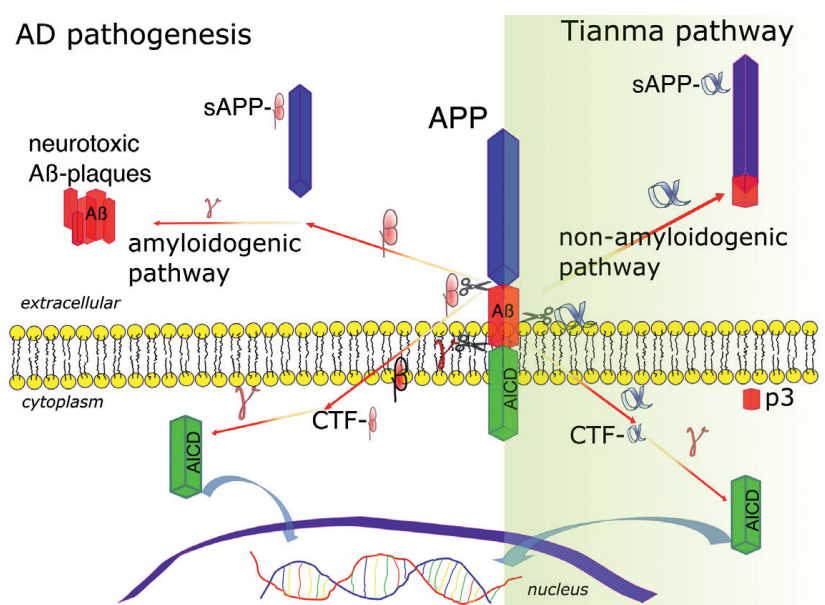

(b)

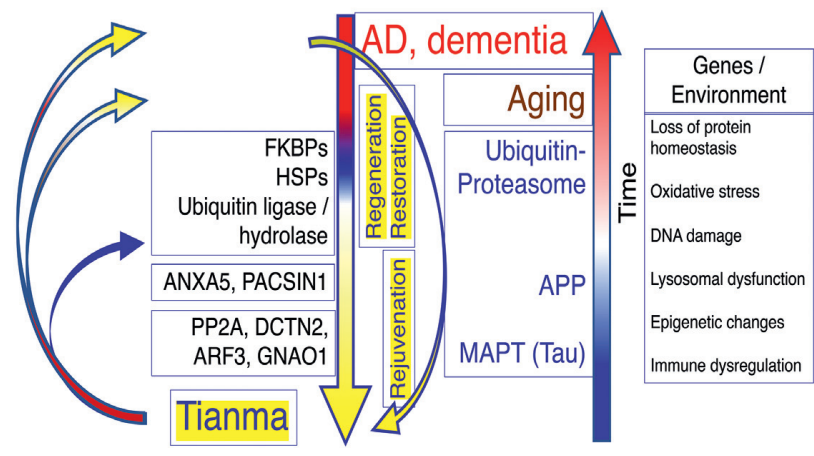

(d)

FIGURE 2: The effects of tianma on AD-related signaling and cognitive function during aging. (a) Qualitative data showing the effects of tianma and other herbs on APP processing. Estimation of soluble sAPP $\alpha$ level in cell culture supernatant (measured by enzyme-linked immunosorbent assay (ELISA)) and relative beta-secretase 1 (BACE1) expression (evaluated by Western blot analyses) of neuronal cells treated with tianma. Neurons were grown and treated with certain concentrations of tianma or other herbs for 24 h. Data are qualitatively represented as relative changes compared with controls. Only tianma-treated neurons showed significant increase of sAPP $\alpha$ level and significantly reduced BACE1 expression $[44,46]$. (b) Schematic overview of the impact of tianma on APP processing. At the measured concentrations, tianma inhibits BACE1, promotes sAPP $\alpha$ production, suppresses amyloid beta peptide (A $\beta$ ) plaque formation, and reduces microtubule-associated protein tau (MAPT) phosphorylation, thereby fostering the nonamyloidogenic pathway [42, 44, 46]. (c) Qualitative data showing the effects of tianma on cognitive function. Tianma enhances memory, learning, and executive function in elderly rats during the Morris water maze, object recognition, and attention set shift tests [32, 41, 43, 46, 49]. (d) Schematic overview of the mechanisms underlying the effects of tianma on dementia. Tianma markedly improves cognitive abilities and protects against aging-related dementia, memory impairment, and neurodegeneration by restoring and rejuvenating cerebrovascular functions in elderly rats $[30,32,33,37,40-44,46,49,72]$.

be increased with a wide range of conditions and lifestyle factors, including global failure of cellular energy metabolism, hypertension, dyslipidemia, hypercholesterolemia, lower physical activity, and poor diet $[22,56,58-66]$.

3.1. Tianma Enhances Acetylcholine- (ACh-) Induced Vasorelaxation, A Measure of the Contractile Force and Elasticity of Aortic Vessels: Vasodilatory Proteomic Profile Changes in Aortic Tissue. Blood vessel tonicity is regulated by vascular smooth muscle cells which modulate contraction and relaxation. Functional aortic tissue proteomic data have demonstrated that long-term treatment with small doses of tianma regulated blood vessel tonicity by mediating the expression of contractile proteins (e.g., actin alpha 2 (ACTA2)) and structural proteins (e.g., desmin (DES), microtubule-associated protein 4 (MAP4), PDZ, and LIM domain 1 (PDLIM1) and vinculin (VCL)), extracellular matrix proteins (ECM, e.g., elastin (ELN), fibulin 5 (FBLN5), and proline- and arginine-rich end leucine-rich repeat protein (PRELP)), and thrombotic proteins (e.g., annexin A2 (ANXA2)), thereby enhancing thoracic aortic contractile force and improving blood vessel elasticity (Figure 1) [67]. Moreover, elevated ANXA2 and reduced level of fatty acid binding protein 4 (FABP4) may prevent atherosclerosis and cardiovascular diseases [68, 69].

By inductive reasoning, tianma could likely prevent many CCVDs, such as headache, hypertension, 
atherosclerosis, and stroke, by facilitating vasodilatory effects that strengthen the arterial structure. Therefore, identification of all the bioactive ingredients in tianma could help facilitate its application as an efficient therapeutic herbal medicine for treatment of CCVDs by elucidating the mechanisms by which it ameliorates these abnormal cardiovascular responses [33, 41, 54, 67, 70, 71].

\section{Tianma Improves Cognitive Function during Aging-Related Dementia}

Accumulating evidence indicates that tianma sharpens several cognitive functions, including memory and learning activities [30, 32, 40, 43, 49]. Moreover, neuroprotective and neuro-regenerative qualities have been attributed to tianma, particularly during aging and aging-related NDs, such as $\mathrm{AD}, \mathrm{PD}$, and $\mathrm{VD}[26,30,36,38-44,47,72]$. Specifically, pharmacologically relevant studies have demonstrated at the cellular and molecular levels that tianma could prevent AD by modulating proteolytic processing of amyloid beta precursor protein (APP), driving the nonamyloidogenic pathway (Figure 2) [41-44, 46].

\section{Discussion}

5.1. Aging and Dementia: Abnormal Protein Structures. In $\mathrm{AD}$, accumulation of $\mathrm{A} \beta$ and hyperphosphorylated MAPT protein act as seeds for prion-like transmission of misfolded proteins to adjacent neurons, where misfolded MAPT further aggregates into neurofibrillary tangles (NFTs) [73-75]. The FKBPs act as a cochaperone in AD brains trying to prevent MAPT degradation by binding to MAPT and increasing its stability via interaction with the peptidylprolyl isomerase (PPIase) domain [76, 77]. However, downregulation of important E3-ligases (tripartite motif containing 32/37 (TRIM32/37)) and chaperone proteins, such as HSPs (e.g., HSP90), might impair hyperphosphorylated MAPT clearance $[20,37]$. HSP90 and STUB1 (STIP1 (stress-induced phosphoprotein 1) homology and U-box containing protein 1, also known as carboxyl terminus of heat shock cognate 70(HSC70-) interacting protein (CHIP)), target hyperphosphorylated MAPT for proteasomal degradation. Hyperphosphorylated MAPT loses its physiological function for axonal transport, aggregates into NFTs, and causes neuron death. In addition, the impaired UPS (consisting of the 26S proteasome, ubiquitin ligases, and ubiquitin hydrolases) and compromised function of HSPs and FKBPs together impair the protein degradation pathway and promote pathophysiological conditions [20, 26, 37].

\subsection{Interference Prevents Protein Misfolding during Aging and} in NDs. The proposed pathomechanism underlying $\mathrm{AD}$ involves $\mathrm{A} \beta$ plaque formation, NFTs, and deregulation of chaperone proteins. Consequently, in AD brains, an impaired UPS system is thought to account for $\mathrm{A} \beta$ aggregation and hyperphosphorylated MAPT-mediated NFT formation, which is potentially furthered by an irregular APP intracellular domain (AICD) signaling pathway [20]. The various protein groups modulated by tianma treatment affect the UPS system, and active tianma ingredients also target molecular chaperones and cochaperones, such as HSPs and FKBP, and modulate the actions of protein phosphate PP2A. Together, these data open new avenues for future investigations into the prophylactic effects of tianma for aging-related dementia and NDs (Figure 2(d)) $[20,26]$.

\section{Conclusion}

The human brain, with its high-level cognitive functions, requires a large degree of flexibility and adaptability for appropriate learning and memory and is very vulnerable to cerebrovascular injuries, such as ischemia or stroke, which can cause NDs and dementia. Tianma has been shown in human clinical studies to be effective against VD [40], and various pre-clinical studies have demonstrated at the molecular and cellular levels its potential as an efficacious anti-aging elixir.

\section{Abbreviations}

$\mathrm{A} \beta$ :

ACh:

ACTA2:

$\mathrm{AD}$ :

AICD:

ANXA2:

APP:

BACE1:

CCVD:

DES:

DM:

ELISA:

ELN:

EOAD:

FABP4:

FBLN5:

FKBP:

G. elata,

tianma:

HSC70:

HSP:

LOAD:

MAP4:

MAPT:

ND:

NFT:

PD:

PDLIM1:

PDZ:

Dlg1:

zo-1:

LIM:

PE:

PPIase:

PRELP.
Amyloid beta peptide

Acetylcholine

Actin alpha 2

Alzheimer's disease

APP intracellular domain

Annexin A2

Amyloid beta precursor protein

Beta-secretase 1

Desmin

Diabetes mellitus

Enzyme-linked immunosorbent assay

Elastin

Early-onset AD

Fatty acid binding protein 4

Fibulin 5

FK506 binding protein

Gastrodia elata Blume

Heat shock cognate 70

Heat shock protein

Late-onset (nonfamilial, sporadic) AD

Microtubule-associated protein 4

Microtubule-associated protein tau

Neurodegenerative disease

Neurofibrillary tangles

Parkinson's disease

PDZ and LIM domain 1

Postsynaptic density protein (PSD95)

Drosophila disc large tumor suppressor

zonula occludens-1 protein

Lin11, Isl-1, Mec-3

Phenylephrine

Peptidylprolyl isomerase
Cerebrocardiovascular disease 
Proline- and arginine-rich end leucine-rich repeat protein

STIP1: $\quad$ Stress-induced phosphoprotein 1

STUB1: $\quad$ STIP1 homology and U-box containing protein 1

TCM: $\quad$ Traditional Chinese medicine

TRIM: $\quad$ Tripartite motif containing

UPS: Ubiquitin proteasome system

VCL: $\quad$ Vinculin

VD: $\quad$ Vascular dementia

WHO: World Health Organization.

\section{Conflicts of Interest}

The author declares no conflicts of interest.

\section{Acknowledgments}

This study was supported by the Basic Science Research Program through the National Research Foundation of Korea (NRF), which was funded by the Ministry of Education (2019R1F1A1056445).

\section{References}

[1] K. Nagata, T. Yamazaki, D. Takano et al., "Cerebral circulation in aging," Ageing Research Reviews, vol. 30, pp. 49-60, 2016.

[2] L. Pini, M. Pievani, M. Bocchetta et al., "Brain atrophy in alzheimer's disease and aging," Ageing Research Reviews, vol. 30, pp. 25-48, 2016.

[3] S. Tarantini, C. H. T. Tran, G. R. Gordon, Z. Ungvari, and A. Csiszar, "Impaired neurovascular coupling in aging and alzheimer's disease: contribution of astrocyte dysfunction and endothelial impairment to cognitive decline," Experimental Gerontology, vol. 94, pp. 52-58, 2017.

[4] M. Vijayan, S. Kumar, J. S. Bhatti, and P. H. Reddy, "Molecular links and biomarkers of stroke, vascular dementia, and alzheimer's disease," Progress in Molecular Biology and Translational Science, vol. 146, pp. 95-126, 2017.

[5] T. Wyss-Coray, "Ageing, neurodegeneration and brain rejuvenation,” Nature, vol. 539, no. 7628, pp. 180-186, 2016.

[6] T. Yang, Y. Sun, Z. Lu, R. K. Leak, and F. Zhang, "The impact of cerebrovascular aging on vascular cognitive impairment and dementia," Ageing Research Reviews, vol. 34, pp. 15-29, 2017.

[7] J. P. da Costa, R. Vitorino, G. M. Silva, C. Vogel, A. C. Duarte, and T. Rocha-Santos, "A synopsis on aging-theories, mechanisms and future prospects," Ageing Research Reviews, vol. 29, pp. 90-112, 2016.

[8] H. Shah, E. Albanese, C. Duggan et al., "Research priorities to reduce the global burden of dementia by 2025," The Lancet Neurology, vol. 15, no. 12, pp. 1285-1294, 2016.

[9] S. Pramanik, Y. A. Sulistio, and K. Heese, "Neurotrophin signaling and stem cells-implications for neurodegenerative diseases and stem cell therapy," Molecular Neurobiology, vol. 54, no. 9, pp. 7401-7459, 2017.

[10] A. M. McGrattan, B. McGuinness, M. C. McKinley et al., "Diet and inflammation in cognitive ageing and alzheimer's disease," Current Nutrition Reports, vol. 8, no. 2, pp. 53-65, 2019.

[11] G. K. Bhatti, A. P. Reddy, P. H. Reddy, and J. S. Bhatti, "Lifestyle modifications and nutritional interventions in aging-associated cognitive decline and alzheimer's disease," Frontiers in Aging Neuroscience, vol. 11, p. 369, 2019.

[12] D. J. Selkoe, “Alzheimer's disease: genes, proteins, and therapy," Physiological Reviews, vol. 81, no. 2, pp. 741-766, 2001.

[13] K. Heese and H. Akatsu, "Alzheimers disease-an interactive perspective," Current Alzheimer Research, vol. 3, no. 2, pp. 109-121, 2006.

[14] R. E. Tanzi and L. Bertram, "New frontiers in alzheimer's disease genetics," Neuron, vol. 32, no. 2, pp. 181-184, 2001.

[15] L. M. Bekris, C.-E. Yu, T. D. Bird, and D. W. Tsuang, "Review article: genetics of alzheimer disease," Journal of Geriatric Psychiatry and Neurology, vol. 23, no. 4, pp. 213-227, 2010.

[16] C. Reitz and R. Mayeux, "Alzheimer disease: epidemiology, diagnostic criteria, risk factors and biomarkers," Biochemical Pharmacology, vol. 88, no. 4, pp. 640-651, 2014.

[17] J. Lindsay, D. Laurin, R. Verreault et al., "Risk factors for alzheimer's disease: a prospective analysis from the Canadian study of health and aging," American Journal of Epidemiology, vol. 156, no. 5, pp. 445-453, 2002.

[18] Y. Ihara, M. Morishima-Kawashima, and R. Nixon, "The ubiquitin-proteasome system and the autophagic-lysosomal system in alzheimer disease," Cold Spring Harbor Perspectives in Medicine, vol. 2, no. 8, p. a006361, 2012.

[19] B. R. Troen, “The biology of aging," Mount Sinai Journal of Medicine, vol. 70, no. 1, pp. 3-22, 2003.

[20] Y. A. Sulistio and K. Heese, "The ubiquitin-proteasome system and molecular chaperone deregulation in alzheimer's disease," Molecular Neurobiology, vol. 53, no. 2, pp. 905-931, 2016.

[21] C. Rowland, The Washington Post, Vol. Business, 209, https:// www.washingtonpost.com/business/economy/alzheimersresearch-is-getting-a-reboot-at-small-companies-focusedon-the-immune-system/2019/07/03/974d8854-91e9-11e9b58a-a6a9afaa0e3e_story.html.

[22] E. Flanagan, D. Lamport, L. Brennan et al., "Nutrition and the ageing brain: moving towards clinical applications," Ageing Research Reviews, vol. 62, Article ID 101079, 2020.

[23] Q. Yan, W. Wang, J. Weng et al., "Dissolving microneedles for transdermal delivery of huperzine A for the treatment of alzheimer's disease," Drug Delivery, vol. 27, no. 1, pp. 1147-1155, 2020.

[24] A. Balan, M. A. Moga, L. Dima, S. Toma, A. Elena Neculau, and C. V. Anastasiu, "Royal jelly-A traditional and natural remedy for postmenopausal symptoms and aging-related pathologies," Molecules, vol. 25, no. 14, p. 3291, 2020.

[25] X. Deng, S. Zhao, X. Liu et al., "Polygala tenuifolia: a source for anti-alzheimer's disease drugs," Pharmaceutical Biology, vol. 58, no. 1, pp. 410-416, 2020.

[26] Y. A. Sulistio and K. Heese, "Proteomics in traditional Chinese medicine with an emphasis on alzheimer's disease," Evidence-Based Complementary and Alternative Medicine, vol. 2015, Article ID 393510, , 2015.

[27] H.-D. Zhan, H.-Y. Zhou, Y.-P. Sui et al., "The rhizome of gastrodia elata blume-an ethnopharmacological review," Journal of Ethnopharmacology, vol. 189, pp. 361-385, 2016.

[28] Y. Shou-Zhong, The Divine Farmer's Materia Medica: A Translation of the Shen Nong Ben Cao Jing, Blue Poppy Press, Boulder, Colo, USA, 2007.

[29] L. Shizhen, Bencao Gangmu: Compendium of Materia Medica, Foreign Language Press, Beijing, China, 2006.

[30] Y. Liu, J. Gao, M. Peng et al., "A review on central nervous system effects of gastrodin," Frontiers in Pharmacology, vol. 9, p. 24, 2018. 
[31] J. C. Heo, S. U. Woo, M. Son et al., "Anti-tumor activity of gastrodia elata blume is closely associated with a GTP-Rasdependent pathway," Oncology Reports, vol. 18, no. 4, pp. 849-853, 2007.

[32] Y.-M. Park, B.-G. Lee, S.-H. Park et al., "Prolonged oral administration of gastrodia elataextract improves spatial learning and memory of scopolamine-treated rats," Laboratory Animal Research, vol. 31, no. 2, pp. 69-77, 2015.

[33] J. W. Xian, A. Y. Choi, C. B. Lau, W. N. Leung, C. F. Ng, and C. W. Chan, "Gastrodia and Uncaria (tianma gouteng) water extract exerts antioxidative and antiapoptotic effects against cerebral ischemia in vitro and in vivo," Chinese Medicine, vol. 11, p. 27, 2016.

[34] L. M. Ojemann, W. L. Nelson, D. S. Shin, A. O. Rowe, and R. A. Buchanan, "Tian ma, an ancient Chinese herb, offers new options for the treatment of epilepsy and other conditions," Epilepsy \& Behavior, vol. 8, no. 2, pp. 376-383, 2006.

[35] X. Xiong, X. Yang, Y. Liu, Y. Zhang, P. Wang, and J. Wang, "Chinese herbal formulas for treating hypertension in traditional Chinese medicine: perspective of modern science," Hypertension Research, vol. 36, no. 7, pp. 570-579, 2013.

[36] A. Manavalan, U. Ramachandran, H. Sundaramurthi et al., "Gastrodia elata blume (tianma) mobilizes neuro-protective capacities," International Journal of Biochemistry and Molecular Biology, vol. 3, no. 2, pp. 219-241, 2012.

[37] A. Manavalan, M. Mishra, L. Feng, S. K. Sze, H. Akatsu, and $\mathrm{K}$. Heese, "Brain site-specific proteome changes in agingrelated dementia," Experimental \& Molecular Medicine, vol. 45, no. 9, p. e39, 2013.

[38] U. Ramachandran, A. Manavalan, H. Sundaramurthi et al., "Tianma modulates proteins with various neuro-regenerative modalities in differentiated human neuronal SH-SY5Y cells," Neurochemistry International, vol. 60, no. 8, pp. 827-836, 2012.

[39] H. Sundaramurthi, A. Manavalan, U. Ramachandran, J.-M. Hu, S. K. Sze, and K. Heese, "Phenotyping of tianmastimulated differentiated rat neuronal b104 cells by quantitative proteomics," Neurosignals, vol. 20, no. 1, pp. 48-60, 2012.

[40] G. Du, K. Chen, W. Zhou et al., "Clinical effect of tianmacuzhi granules on senile vascular dementia," Zhongguo Zhong Yao Za Zhi Zhongguo Zhongyao Zazhi China Journal of Chinese Materia Medica, vol. 23, no. 11, pp. 695-698, 1998.

[41] R. Shi, C. B. Zheng, H. Wang et al., "Gastrodin alleviates vascular dementia in a 2-VO-vascular dementia rat model by altering amyloid and tau levels," Pharmacology, vol. 105, no. 78, pp. 386-396, 2020.

[42] M. Li and S. Qian, "Gastrodin protects neural progenitor cells against amyloid $\beta$ (1-42)-induced neurotoxicity and improves hippocampal neurogenesis in amyloid $\beta$ (1-42)-injected mice," Journal of Molecular Neuroscience, vol. 60, no. 1, pp. 21-32, 2016.

[43] Y. Hu, C. Li, and W. Shen, "Gastrodin alleviates memory deficits and reduces neuropathology in a mouse model of alzheimer's disease," Neuropathology: Official Journal of the Japanese Society of Neuropathology, vol. 34, no. 4, pp. 370377, 2014.

[44] J.-S. Zhang, S.-F. Zhou, Q. Wang et al., "Gastrodin suppresses BACE1 expression under oxidative stress condition via inhibition of the PKR/eIF $2 \alpha$ pathway in alzheimer's disease," Neuroscience, vol. 325, pp. 1-9, 2016.

[45] G. B. Huang, T. Zhao, S. S. Muna et al., "Therapeutic potential of Gastrodia elata Blume for the treatment of alzheimer's disease," Neural Regeneration Research, vol. 8, no. 12, pp. 1061-1070, 2013.

[46] M. Mishra, J. Huang, Y. Y. Lee et al., "Gastrodia elata modulates amyloid precursor protein cleavage and cognitive functions in mice," Bioscience Trends, vol. 5, no. 3, pp. 129-138, 2011.

[47] L. F. Liu, J. X. Song, J. H. Lu et al., "Tianma Gouteng Yin, a Traditional Chinese Medicine decoction, exerts neuroprotective effects in animal and cellular models of Parkinson's disease," Scientific Reports, vol. 5, Article ID 16862, 2015.

[48] Y. Y. Huang, L. F. Liu, R. Q. Yue et al., "Full component analysis of Tianma-Gouteng-Yin," Chinese Medicine, vol. 11, p. 44, 2016.

[49] M.-T. Hsieh, C.-R. Wu, and C.-F. Chen, "Gastrodin and p-hydroxybenzyl alcohol facilitate memory consolidation and retrieval, but not acquisition, on the passive avoidance task in rats," Journal of Ethnopharmacology, vol. 56, no. 1, pp. 45-54, 1997.

[50] C.-J.-S. Lai, Y. Yuan, D.-H. Liu et al., "Untargeted metabolite analysis-based UHPLC-Q-TOF-MS reveals significant enrichment of p-hydroxybenzyl dimers of citric acids in fresh beige-scape Gastrodia elata (Wutianma)," Journal of Pharmaceutical and Biomedical Analysis, vol. 140, pp. 287-294, 2017.

[51] L.-C. Lin, Y.-F. Chen, W.-C. Lee, Y.-T. Wu, and T.-H. Tsai, "Pharmacokinetics of gastrodin and its metabolite p-hydroxybenzyl alcohol in rat blood, brain and bile by microdialysis coupled to LC-MS/MS," Journal of Pharmaceutical and Biomedical Analysis, vol. 48, no. 3, pp. 909-917, 2008.

[52] M. Li, Y. Du, L. Wang et al., "Efficient discovery of quality control markers for gastrodia elata tuber by fingerprint-efficacy relationship modelling," Phytochemical Analysis, vol. 28, no. 4, pp. 351-359, 2017.

[53] J. H. Ha, D. U. Lee, J. T. Lee et al., "4-Hydroxybenzaldehyde from Gastrodia elata B1. is active in the antioxidation and GABAergic neuromodulation of the rat brain," Journal of Ethnopharmacology, vol. 73, no. 1-2, pp. 329-333, 2000.

[54] E. Picano, R. M. Bruno, G. F. Ferrari, and U. Bonuccelli, "Cognitive impairment and cardiovascular disease: so near, so far," International Journal of Cardiology, vol. 175, no. 1, pp. 21-29, 2014.

[55] A. M. Hooghiemstra, A. S. Bertens, A. E. Leeuwis et al., "The missing link in the pathophysiology of vascular cognitive impairment: design of the heart-brain study," Cerebrovascular Diseases Extra, vol. 7, no. 3, pp. 140-152, 2017.

[56] U. Kumari and K. Heese, "Cardiovascular dementia-a different perspective," The Open Biochemistry Journal, vol. 4, pp. 29-52, 2010.

[57] R. A. L. De Sousa, A. R. Harmer, D. A. Freitas, V. A. Mendonça, A. C. R. Lacerda, and H. R. Leite, "An update on potential links between type 2 diabetes mellitus and alzheimer's disease," Molecular Biology Reports, vol. 47, no. 8, pp. 6347-6356, 2020.

[58] J. Leszek, E. V. Mikhaylenko, D. M. Belousov et al., "The links between cardiovascular diseases and alzheimer's disease," Current Neuropharmacology, 2020, https://www.eurekaselect.com/184274/article.

[59] A. Datta, J. E. Park, X. Li et al., "Phenotyping of anin vitromodel of ischemic penumbra by iTRAQ-based shotgun quantitative proteomics," Journal of Proteome Research, vol. 9, no. 1, pp. 472-484, 2010.

[60] A. Datta, Q. Jingru, T. H. Khor, M. T. Teo, K. Heese, and S. K. Sze, "Quantitative neuroproteomics of anIn vivorodent 
model of focal cerebral ischemia/reperfusion injury reveals a temporal regulation of novel pathophysiological molecular markers," Journal of Proteome Research, vol. 10, no. 11, pp. 5199-5213, 2011.

[61] A. Datta, H. Akatsu, K. Heese, and S. K. Sze, "Quantitative clinical proteomic study of autopsied human infarcted brain specimens to elucidate the deregulated pathways in ischemic stroke pathology," Journal of Proteomics, vol. 91, pp. 556-568, 2013.

[62] A. Ahmad, V. Patel, J. Xiao, and M. M. Khan, "The role of neurovascular system in neurodegenerative diseases," Molecular Neurobiology, vol. 57, no. 11, pp. 4373-4393, 2020.

[63] Z. Bartochowski, J. Conway, Y. Wallach, B. Chakkamparambil, S. Alakkassery, and G. T. Grossberg, "Dietary interventions to prevent or delay alzheimer's disease: what the evidence shows," Current Nutrition Reports, vol. 9, no. 3, pp. 210-225, 2020.

[64] A. C. van den Brink, E. M. Brouwer-Brolsma, A. A. M. Berendsen, and O. van de Rest, "The mediterranean, dietary approaches to stop hypertension (DASH), and mediterranean-DASH intervention for neurodegenerative delay (MIND) diets are associated with less cognitive decline and a lower risk of alzheimer's disease-a review," Advances in Nutrition, vol. 10, no. 6, pp. 1040-1065, 2019.

[65] F. Pistollato, R. C. Iglesias, R. Ruiz et al., "Nutritional patterns associated with the maintenance of neurocognitive functions and the risk of dementia and Alzheimer's disease: a focus on human studies," Pharmacological Research, vol. 131, pp. 3243, 2018

[66] V. Solfrizzi, C. Custodero, M. Lozupone et al., "Relationships of dietary patterns, foods, and micro- and macronutrients with alzheimer's disease and late-life cognitive disorders: a systematic review," Journal of Alzheimer's Disease, vol. 59, no. 3, pp. 815-849, 2017.

[67] L. Feng, A. Manavalan, M. Mishra, S. K. Sze, J.-M. Hu, and K. Heese, "Tianma modulates blood vessel tonicity," The Open Biochemistry Journal, vol. 6, pp. 56-65, 2012.

[68] E. C. Flood and K. A. Hajjar, "The annexin A2 system and vascular homeostasis," Vascular Pharmacology, vol. 54, no. 36, pp. 59-67, 2011.

[69] M. Furuhashi and G. S. Hotamisligil, "Fatty acid-binding proteins: role in metabolic diseases and potential as drug targets," Nature Reviews Drug Discovery, vol. 7, no. 6, pp. 489-503, 2008.

[70] I. Ferrer, "Cognitive impairment of vascular origin: neuropathology of cognitive impairment of vascular origin," Journal of the Neurological Sciences, vol. 299, no. 1-2, pp. 139-149, 2010.

[71] T. Tarumi and R. Zhang, "Cerebral blood flow in normal aging adults: cardiovascular determinants, clinical implications, and aerobic fitness," Journal of Neurochemistry, vol. 144, no. 5, pp. 595-608, 2018.

[72] A. Manavalan, L. Feng, S. K. Sze, J.-M. Hu, and K. Heese, "New insights into the brain protein metabolism of gastrodia elata-treated rats by quantitative proteomics," Journal of Proteomics, vol. 75, no. 8, pp. 2468-2479, 2012.

[73] D. M. Walsh and D. J. Selkoe, "Amyloid $\beta$-protein and beyond: the path forward in alzheimer's disease," Current Opinion in Neurobiology, vol. 61, pp. 116-124, 2020.

[74] A. A. Mamun, M. S. Uddin, B. Mathew, and G. M. Ashraf, "Toxic tau: structural origins of tau aggregation in alzheimer's disease," Neural Regeneration Research, vol. 15, no. 8, pp. 1417-1420, 2020.
[75] S. A. Kent, T. L. Spires-Jones, and C. S. Durrant, "The physiological roles of tau and $\mathrm{A} \beta$ : implications for alzheimer's disease pathology and therapeutics," Acta Neuropathologica, vol. 140, no. 4, pp. 417-447, 2020.

[76] L. J. Blair, J. D. Baker, J. J. Sabbagh, and C. A. Dickey, "The emerging role of peptidyl-prolyl isomerase chaperones in tau oligomerization, amyloid processing, and alzheimer's disease," Journal of Neurochemistry, vol. 133, no. 1, pp. 1-13, 2015.

[77] G. Caminati and P. Procacci, "Mounting evidence of FKBP12 implication in neurodegeneration," Neural Regeneration Research, vol. 15, no. 12, pp. 2195-2202, 2020. 